\title{
Developing a cloud virtual maintenance system for machine tools management
}

\author{
Yueh-Ling Lin*, Chih-Chieh Lin, and Hung-Sheng Chiu \\ Central Industry Research \& Service Division, CID, \\ Institute for Information Industry, \\ 7 Guangming Road, Chung Hsing New Village, \\ Nantou City, Nantou County, Taiwan 54071, ROC \\ yuehlin@iii.org.tw erir@iii.org.tw bbchiu@iii.org.tw
}

\begin{abstract}
Developing efficient maintenance system for Taiwan's machine-tool industry is important because of the cloud service enhancement recently. This study provides innovative maintenance system via cloud virtual computing for machine tool management. The virtual maintenance cloud is a monitoring system for precision manufacturing plant. The virtual maintenance cloud can combine eight kinds of numerical controllers following TMTC (Taiwan MTConnect Protocol). It is compatible with MTConnect function to build the client monitoring application services for the whole plant. The system provides 3 ways to determine the tool life cycle including tool usage frequency, tool usage time, and tool usage distance. A remote control platform was developed for controlling specific number of tools. The system can extract numerical controller data across various brands to achieve the whole plant monitoring. The proposed system was demonstrated by following TMTC communication protocol to develop tool management and application services for machine tool industry. The tool life management system can enhance information used for tool usage analysis for manufacturing machine tools. Thus, the system can increase tool usage rate, decrease processing time, and reduce waste of workpiece materials.
\end{abstract}

Keywords-virtual maintenance cloud; tool life management; TMTC (Taiwan MTConnect Protocol); machine tools management

\section{INTRODUCTION}

There are various brands of numerical controllers. However, these controllers do not have a common data access method [1]. It results in difficult to build the monitoring system for the whole plant [2]. Virtual Maintenance Cloud extracts data by ServBox [3]. It transmitted network packets to communicate with the numerical controller via Ethernet interface. It provides more than thirteen thousand kinds of parameters from FANUC controller. It covers $80 \%$ of the numerical control brands [4]. Developers can get the numerical controller parameters to develop customized applications and services with TMTC communication protocol [5].

Virtual Maintenance Cloud as a remote control platform collected different data from plants [6]. Users only need to log into the system through a browser to master the information collected from different machinery equipment in different plants. It provides user access management, application services list, and language switching functions [7]. The current system has build some application service including real-time status monitor, machine alarm, NC program management, Machine Availability, and tool management [8].

For common medium and big plants, IT system is used for the tool management because of the large number of tools [9]. Due to various kinds of tools, some specific tools are expensive [10]. The IT system can help increase the efficiency of tool management. The more difficult part of the technology is to get the correct tool replace time. The correct analysis of the remaining tool life helps users replace tools in advance. The system can not only extract relevant tool data from front sensing system, but also help monitor tool data similar to common sensing system. It helps avoid additional costs caused by earlier tool replace and tool usage dropping. It also helps avoid additional cost caused by later tool replace and tool broken as well as workpiece waste, processing time waste, and machine damage.

The tool management system aims to help manufacturing plants for the whole plant tool management. The system can extract data including historical information and real-time information for further analysis of the remaining tool life. Users can explicitly get the tool replace time and improve the tool usage. To achieve this goal, the system provides 3 ways to determine the tool life cycle including tool usage frequency, tool usage time, and tool usage distance.

\section{METHOD}

\section{A. Virtual Maintenance Cloud}

Virtual Maintenance Cloud is a monitoring system for precision manufacturing plant. By introducing ICT technology, it makes mechanical equipment have networking capabilities through crossing devices. It tandems all the machine tool equipment and controls the whole plant tools in the whole plant. The system is a sensing system for online application service as shown in Figure 1. The system provides tools used for processing management by manufacturing factories. Figure 2 shows the permission page where users are authorized to access. Users can identify the number of the tool used by the factories. 
Users can keep the remaining life for tool usage with the online virtual maintenance system.

\section{B. Tool Management System}

Virtual Maintenance Cloud can perform monitoring in the entire manufacturing plant. It extracts numerical controller data through cross brands. The system has been applied to manage tools in the entire plant. The tool management system is only used for controlling and validating specific numbers of tools. The tool life analysis method of the system is based on the virtual maintenance software. Figure 3 shows the machine tools management selection page. Users can save additional cost to purchase hardware. As for the licensing price and technology transfer fee, they are based on the following information.

- The number of the tools

- $\quad$ The number of the machines

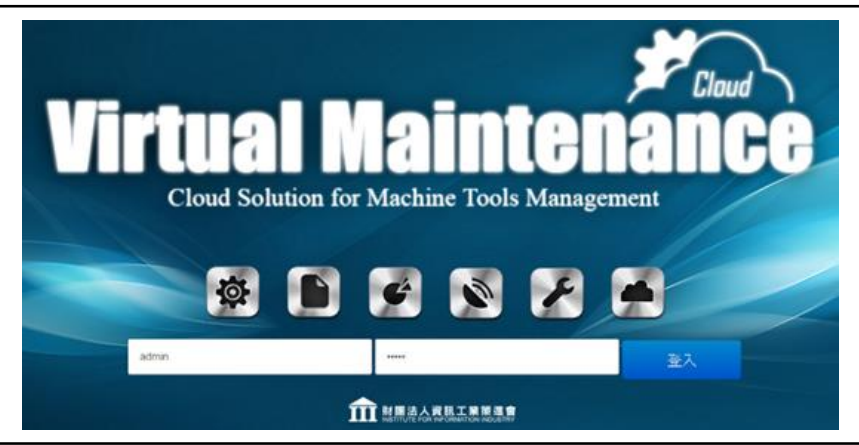

Figure 1. The login page of the Virtual Maintenance Cloud. Users can login through user account and password. cycle management can immediate feedback information can inform users to replace tools at correct time. As a result, the system can increase tool usage, decrease processing time, and reduce material waste. The tool management in the whole plant can master the operation status of the factory equipment by the extraction function from the cross-brands numerical controller. It develops various applications rapidly. It can increase the tool operation efficiency in plant. The concepts of the 2 services are as followings:

- For tool life cycle management:

Conduct majorly for the tool replace time according to 3 conditions including processing times, production time, and processing distance to manage the tool life. It helps avoid additional cost caused by earlier tool replace and tool usage dropping. It also helps avoid additional cost caused by later tool replace and tool broken as well as workpiece waste, processing time waste, and machine damage.

- For tool data management:

Conduct the tool data management for the entire tool life cycle including static tool data and dynamic tool data. Static tool data includes tool inventory, tool storage records, tool position, and tool correction table in query and update results. Dynamic tool data includes tool condition monitoring, machine condition monitoring, processing program monitoring during machine operation. In this way, the plant managers can get the overall tool data for decision making.

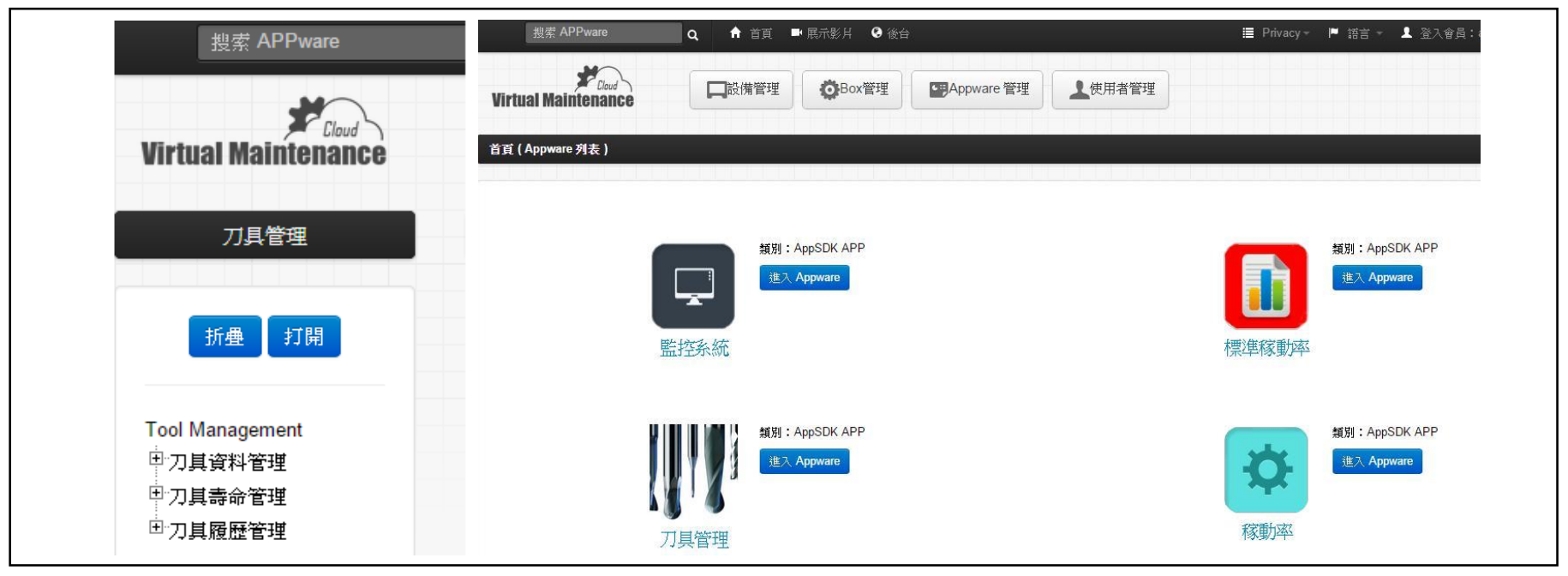

Figure 2. Application Service Selection List. Application Service APP List.

\section{Services provided by the Virtual Maintenance Cloud}

Virtual Maintenance Cloud can provide 2 services for users. One of the system is for machine tools management, and the other of the system is for plant monitoring and management. It not only can help users fully control the tool conditions, but also can increase the tool operation efficiency in plant. Tool life
- $\quad$ For factory monitoring and management:

By cross-brand controller detection technology, it controls the whole plant operational status. It currently supports eight kinds of controllers, including FANUC, Mitsubishi, Siemens, Heidenhain, Hurco, Syntec, Intek, LNC. It covers $80 \%$ of the numerical controllers which constructing the whole plant monitoring system, as listed in table I below. It translates into TMTC communication protocol as a standard for client

Identify applicable sponsor/s here. (sponsors) 
application development. The full name of TMTC is Taiwan MTConnect referred to MTConnect [11]. It divides the parameters of the numerical controller by hierarchical architecture. In order to increase the flexibility of the monitoring system, it added parameters writeback feature. Virtual Maintenance Cloud provides TMTC application services, including real-time monitoring, machine warning, machining process management, tool management, and overall equipment efficiency. It can be applied to control the state of machine operation, the product quality, the efficiency of searching tools, and overall plant operational efficiency.

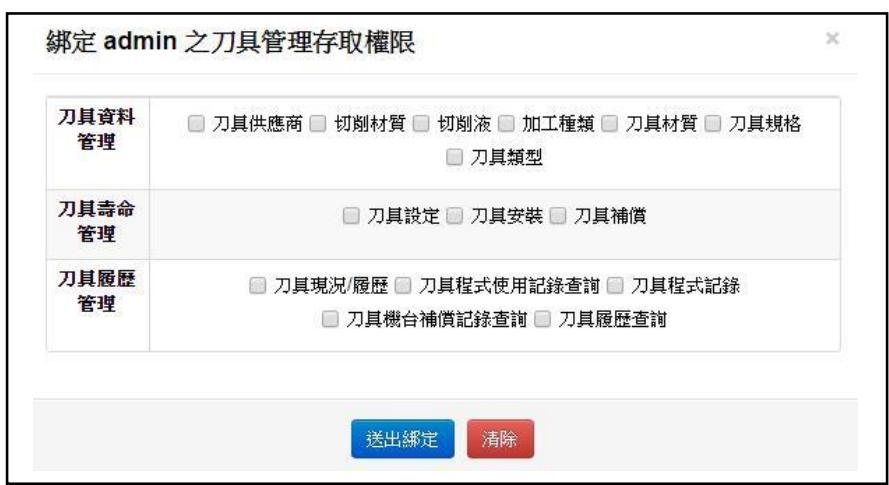

Figure 3. The permission page for users to set access authorities to query the data.

\section{Methods to Determine the Tool Life Cycle}

Virtual Maintenance Cloud extracts data by ServBox. It transmitted network packets to communicate with the numerical controller via Ethernet interface. This machine tool data acquisition device and method won the Gold medal Award of 2014 Taipei International invention show \& technomart News as shown in Figure 4 [12]. It provides more than thirteen thousand kinds of parameters from FANUC controller. It covers $80 \%$ of the numerical control brands. Developers can get the numerical controller parameters to develop customized applications and services with TMTC communication protocol. To achieve this goal, the system provides 3 ways to determine the tool life cycle including tool usage frequency, tool usage time, and tool usage distance.

For the first way, tool usage frequency represents the total number of time of tool to be used in the process. It's practical and intuitive for users, and it's used by most manufacturers. However, the weakness is various processing time in each production. Most errors occur in tool life management.

For the second way, tool usage time represents the total amount of time of tool to be used in the process. It's necessary to extract data from the $\mathrm{CNC}$ controller for further analysis. In this way, the tool life prediction is much more accuracy than the tool usage frequency. However, the weakness is various processing speed in each production. Still, some errors occur and affect the accuracy of tool life prediction.

For the third way, tool usage distance represents the actual distance between tool and workpiece in the process. Overall, it's the most accurate method among the 3 ways. It's necessary to extract exact tool distance data from the CNC controller. By multiplying the server feeding time and cutting time, the value of tool wastage in per minute can be obtained. Moreover, it helps predict the remaining tool life. In this way, the system will continue to extract data from machine during operation. Once the system detects the tool life is coming to end, an alarm message will generate to warn users to replace a new tool.

TABLE I. EIGHT KINDS OF NUMERICAL CONTROLLERS FOLLOWING TMTC (TAIWAN MTCONNECT PROTOCOL)

\begin{tabular}{|c|c|c|}
\hline Item & Brand & Model Type \\
\hline 1 & FANUC & $\begin{array}{c}\text { i-series with Ethernet } \\
(0 \mathrm{i} \cdot 16 \mathrm{i} \cdot 21 \mathrm{i} \cdot 30 \mathrm{i} \cdot 31 \mathrm{i})\end{array}$ \\
\hline 2 & Mitsubishi & M70 $、$ M700 \\
\hline 3 & Siemens & $\begin{array}{c}840 \mathrm{D} \text { SL with RPC } \\
\text { 6FC8500-OAP50-OYBO })\end{array}$ \\
\hline 4 & Heidenhain & iTNC530 enabled DNC \\
\hline 5 & Hurco & NODE-600 \\
\hline 6 & Syntec & EZ Series \\
\hline 7 & Intek & M670 \\
\hline 8 & LNC & GNC6000 \\
\hline
\end{tabular}

\section{RESULTS AND DISCUSSIONS}

Every tool has its own tool life, so it is impossible to predict the life of each tool accurately. Tool life of different specifications can be collected through long-time experience. The recommended tool life can be provided by the tool manufacturer and machinery plant. The tool management system as shown in Figure 5 8 provides users to set up tool life. The method to efficiently determine the tool life cycle includes tool usage frequency, tool usage time, and tool usage distance. The most accurate is the tool usage distance. Tool life management system performs tool usage frequency, tool usage time, and tool usage distance in practice. The best efforts can improve tool usage.

For product comparison, this study supplies a table showing how the proposed product compares to the existing products. Table $\Pi$ shows a comparison between products of tool life management system from RenAn Information company [13] and cyber production center from Mazak company [14]. In addition, it illustrates how the proposed product improves on the competitive products. Institute for Information Industry (III) is an official R\&D unit and non-profit group. The institute provides professional IT services through technology transfer to help cooperation and companies in Taiwan. With the mature technology, it helps establish a spin-off company to provide innovative IT technology in Taiwan. The R\&D team in Institute for Information Industry (III) helps the manufacturing companies improve the overall equipment efficiency (OEE). The development of the tool management system helps companies in Taiwan increase the efficiency of tool management.

Identify applicable sponsor/s here. (sponsors) 


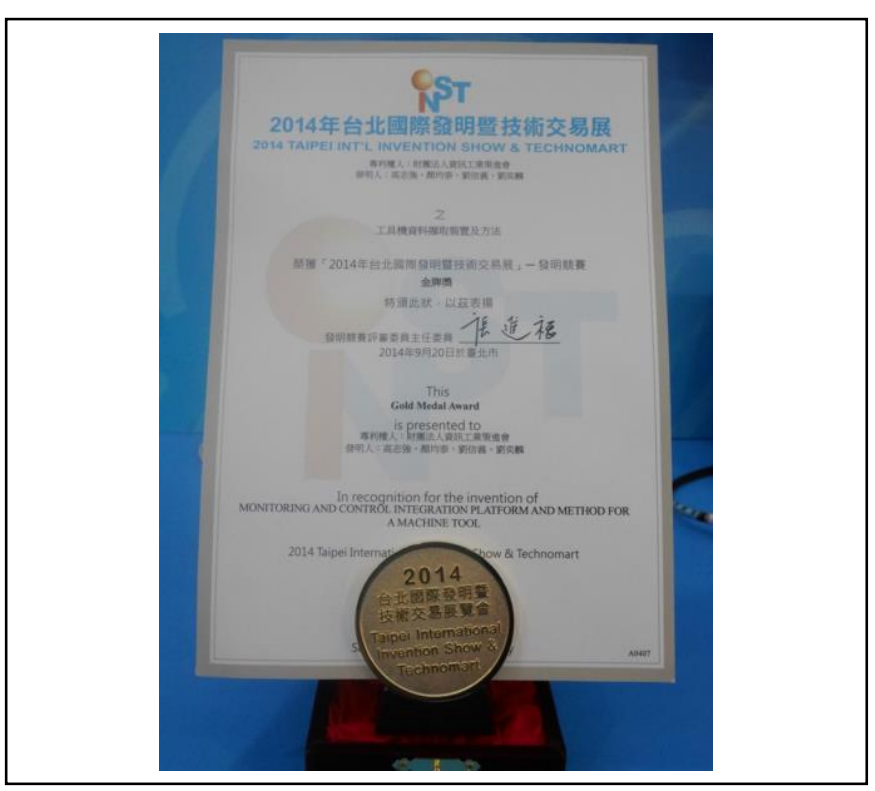

Figure 4. Machine tool data acquisition device and method won the Gold medal Award of 2014 Taipei International invention show \& technomart News $[14,15]$.

\section{CONCLUSION}

Virtual Maintenance Cloud can combine eight kinds of numerical controllers following TMTC (Taiwan MTConnect Protocol). It is compatible with MTConnect write function. It helps build the client monitoring application services for the whole plant. Due to many conditions can affect the tool life, the 3 main methods to efficiently determine the tool life cycle including tool usage frequency, tool usage time, and tool usage distance. The system provides not only tool usage frequency, but also tool usage time and tool usage distance to help predict the remaining tool life more accurately. It helps manufacturers avoid additional cost caused by purchasing other tool measurements.

Tool life management system provides plant manager a convenient way to get tool data easily. On the one hand, it helps increase the tool operation efficiency in plant. On the other hand, it helps avoid additional production cost. The system considers the whole tool life cycle management including tool buy, tool receive, tool install, tool use, tool replace, etc. For the large number of tools, the system helps improve the overall equipment efficiency (OEE) in plant to manage various kinds of expensive tools.

Tool life management system implements three determination ways. The innovation process achieves the determination way of tool life distance. The processing distance is calculated by multiple controller analysis. It avoids error caused by cutting tool speed. It does not require additional approximately \$100,000 tool measuring instruments and effectively reduces the expensive cost.

\section{ACKNOWLEDGMENT}

This study is conducted under the "2015 Project of Development of Industrial Platform for Value-Added Service via Intelligent Sensing Technology (2/4)" of the Institute for Information Industry which is subsidized by the Ministry of Economy Affairs of the Republic of China.

\section{REFERENCES}

[1] A. Gupta and V. U. Srinivasa, "Black Box for Machine Tools: Based on Open CNC Architecture Control Systems," ACEEE Int. J. on Control System and Instrumentation, vol. 3, no. 1, 2012, pp. 6-10.

[2] Q. Ning, T. Wang, L. Liu, and Y. Jiang, "Research on CNC Machine Tools Integration Condition Monitoring System and Its Information Exchange Technology," Future Computing, Communication, Control and Management, Lecture Notes in Electrical Engineering, vol. 144, 2012, pp. 441-450.

[3] Precision Machinery Service Development: Machine Tool Solution and Service Verification, Ministry of Economic Affairs in Taiwan, Annual Report, 2014.

[4] ICT Standards and Intelligent Service Platform Development, Ministry of Economic Affairs in Taiwan, Annual Report, 2013.

[5] ICT Standards and Intelligent Service Platform Development, Ministry of Economic Affairs in Taiwan, Annual Report, 2013, pp. 54-55.

[6] F. Tao, Y. Cheng, D. L. Xu, and L. Zhang, "CCIoT-CMfg: Cloud Computing and Internet of Things-Based Cloud Manufacturing Service System," IEEE Transactions on Industrial Informatics, vol. 10, issue 2, 2014, pp. 1435-1442.

[7] Industry Intelligent Sensing Service Platform Development, Ministry of Economic Affairs in Taiwan, Midterm Report, 2014, pp. 47.

[8] L. Zhang, Y. Luo, F. Tao, B. H. Lia, L. Ren, X. Zhang, H. Guo, Y. Cheng, A. Hu, and Y. Liu, "Cloud manufacturing: a new manufacturing paradigm," Enterprise Information Systems, vol. 8, issue 2, 2014, pp. 167-187.

[9] Z. Bi, L. D. Xu, and C. Wang, "Internet of Things for Enterprise Systems of Modern Manufacturing," IEEE Transactions on Industrial Informatics, vol. 10, issue 2, 2014, pp. 1537-1546.

[10] Solution Architecture for Industry 4.0, BOSCH, 2013.

[11] MTConnect Institute, MTConnect-OPC UA Companion Specification DRAFT Version 1.0, 2011, pp. 12-20.

[12] Yueh-Ling Lin, Chih-Chieh Lin, and Hung-Sheng Chiu. "The Development of Intelligent Service System for Machine Tool Industry The Comparison of Taiwan Machine Tool Connect (TMTC) Standard with the MTConnect Standard", IEEE International Conference on Industrial Networks and Intelligent Systems (IEEE INISCom), Tokyo, Japan, March 2-4, 2015.

[13] http://www.renan.com.tw

[14] http://www.mazak.eu/Cyber-Production-Center

[15] Chih-Chieh Lin, Chun-Tai Yen, and Hung-Sheng Chiu. Machine tool data acquisition device and method, I426364, ROC. 


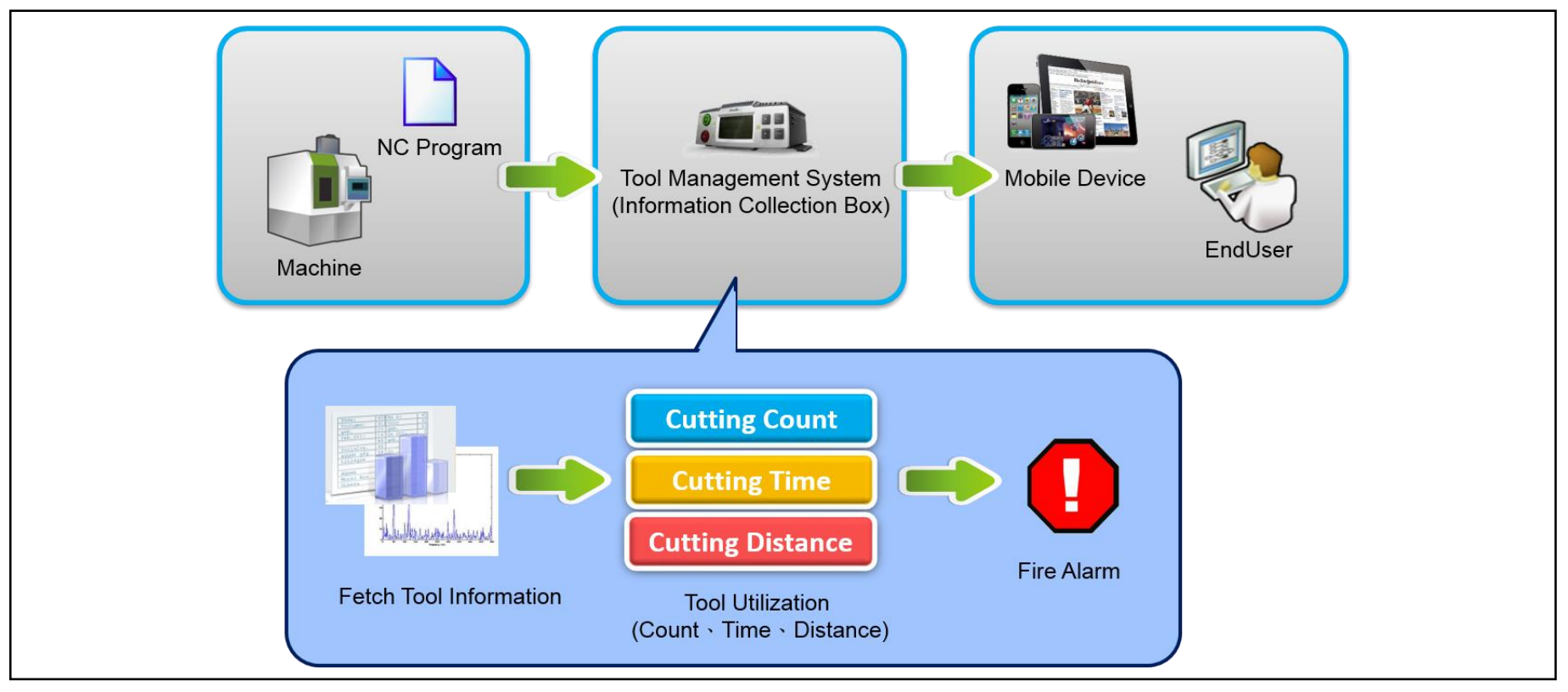

Figure 5. Tool Life Cycle Management

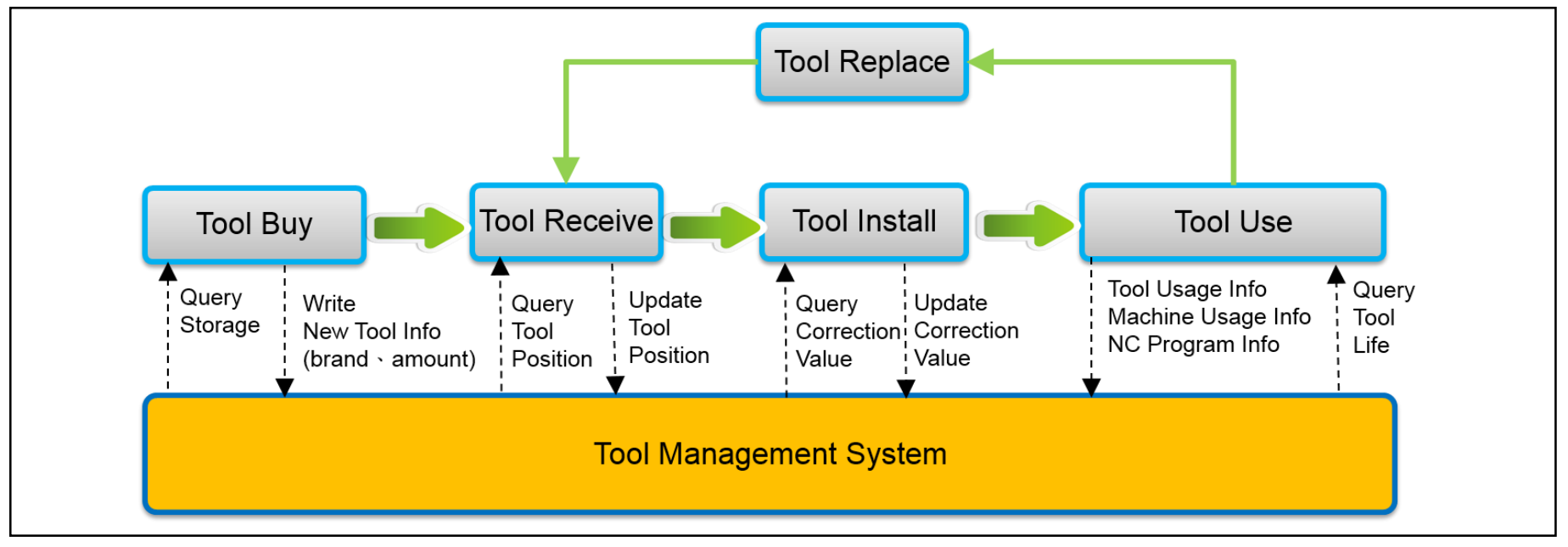

Figure 6. Tool Life Analysis Process 


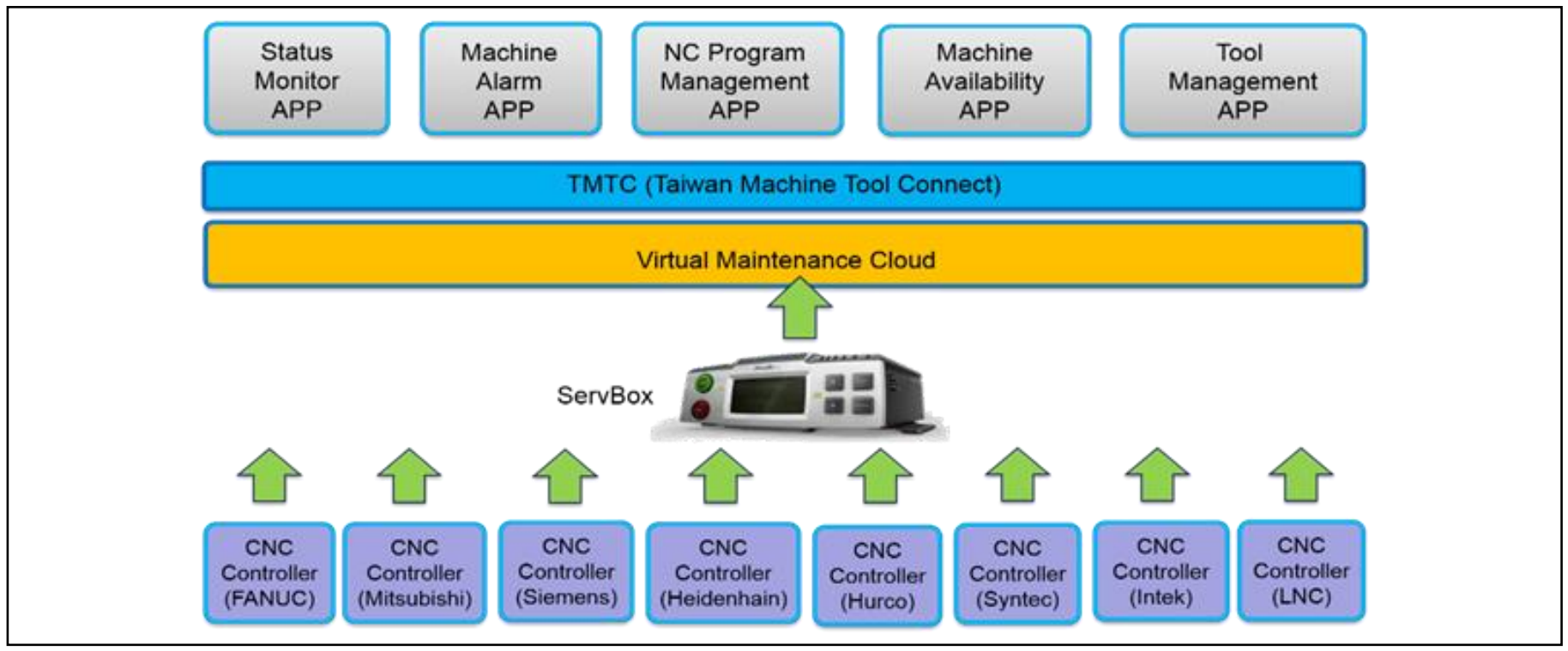

Figure 7. The structure of the virtual maintenance cloud.

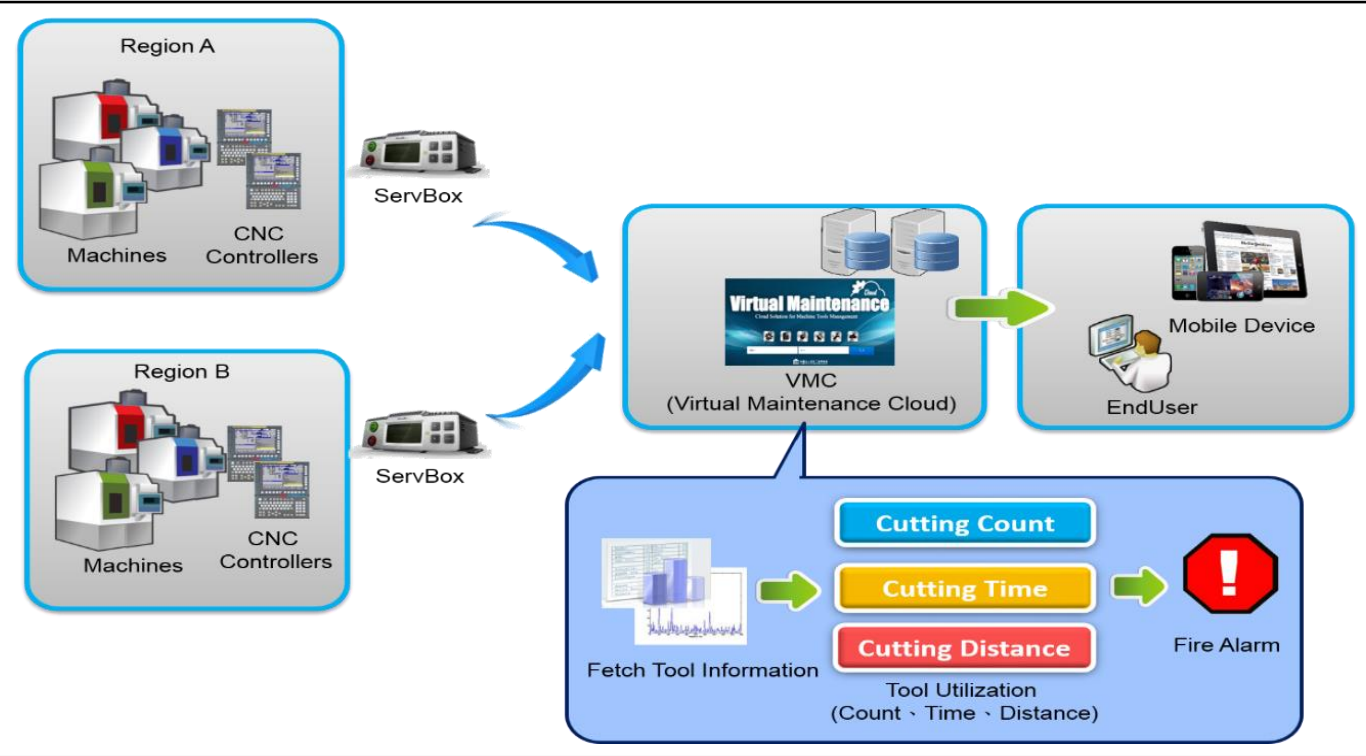

Figure 8. The framework for tool life analysis process. 


\begin{tabular}{|c|c|c|c|}
\hline Item & $\begin{array}{l}\text { Virtual Maintenance Cloud of } \\
\text { Institute for Information Industry (III) }\end{array}$ & Mazak Cyber Production Center & $\begin{array}{c}\text { RenAn Information } \\
\text { (TLM ; Tool Life Management System) }\end{array}$ \\
\hline Management in the Entire Plant & * With cross-brands numerical controller (8 kinds) & * Without cross-brands numerical controller & * With cross-brands numerical controller (5 kinds) \\
\hline Communication protocol & $\begin{array}{l}* \text { With TMTC communication protocol (Write- } \\
\text { back function with parameters) }\end{array}$ & $\begin{array}{l}* \text { With MTConnect communication protocol } \\
\text { (Without write-back function with parameters) }\end{array}$ & * No standard communication protocol \\
\hline Tool Life Analysis Method & $\begin{array}{l}* 3 \text { ways to determine the tool life cycle including } \\
\text { tool usage frequency, tool usage time, and tool } \\
\text { usage distance. }\end{array}$ & $\begin{array}{l}\text { * Support } 1 \text { way to determine the tool life: } \\
\text { processing time }\end{array}$ & $\begin{array}{l}\text { * Support } 1 \text { way to determine the tool life: } \\
\text { processing time }\end{array}$ \\
\hline
\end{tabular}

